\title{
TIDAL DWARF GALAXIES
}

\author{
P.-A. DUC \\ ESO, Karl-Schwarzschild Straße 2 \\ D-85748 Garching bei München, Germany \\ I.F. MIRABEL \\ CEA, SAp, C.E. Saclay \\ 91191 Gif-sur-Yvette Cedex, France \\ AND \\ E. BRINKS \\ Departamento de Astronomia, Univ. de Guanajuato \\ Apdo. Postal 144, Guanajuato, Gto. 36000, Mexico
}

\section{Introduction}

The life and evolution of galaxies are dramatically affected by environmental effects. Interactions with the intergalactic medium and collisions with companions cause major perturbations in the morphology and contents of galaxies: in particular stars and gas clouds may be gravitationally pulled out from their parent galaxies during tidal encounters, forming rings, tails and bridges. This debris of collisions lies at the origin of a new generation of small galaxies, the so-called "tidal dwarf galaxies" (hereafter TDGs). Such an exotic way of forming galaxies was put forward by Schweizer (1978) and by Mirabel et al. (1992), who clearly observed the genesis of a star-forming object, out of material tidally expelled from the interacting system NGC 4038/39 ("The Antennae"). Recent studies, based on optical and HI observations, have shown that TDGs actually form a class of "recycled" objects with some properties similar to the more classical dwarf irregulars (dIrr) and blue compact dwarf galaxies (BCDs).

\section{Case studies}

Fig. 1 presents different examples of interactions: spiral-spiral collisions (NGC 4038/39), merger between spirals (NGC 7252) and encounters involving early-type galaxies (Arp 105, "The guitar galaxy"; NGC 5291, "the seashell galaxy"). Long tidal tails are clearly seen emanating from the parent galaxies. At their tip, at distances up to $100 \mathrm{kpc}$ from the nuclei, small irregular objects are found with absolute magnitudes typical of dwarf galaxies. They host blue compact clumps that we identified with star-forming regions. Their spectra show emission lines typical of HII regions, ionized by massive OB stars younger than $10 \mathrm{Myrs}$. Given the time scale for the formation of clumps in tidal tails - typically $1 \mathrm{Gyr}$-, the stars at the tip of the antennae must have been born in situ. The contours of the HI column density are superimposed on the CCD images. It is striking on these images that the central regions of the parent galaxies contain little atomic gas, whereas the optical tails, and especially the tidal dwarfs at their tip, are associated with HI clouds as massive as $5 \times 10^{9} \mathrm{M}_{\odot}$ (see also Hibbard \& van Gorkom, 1996).

\section{Properties of tidal dwarf galaxies}

We have carried out multi-wavelength observations to study the properties of about 20 TDGs. Optical imaging and spectroscopy were obtained with the CFHT at Mauna Kea and the NTT at la Silla; near-infrared imaging was performed at the ESO/MPI $2.2 \mathrm{~m}$ and HI observations were obtained at the VLA. 


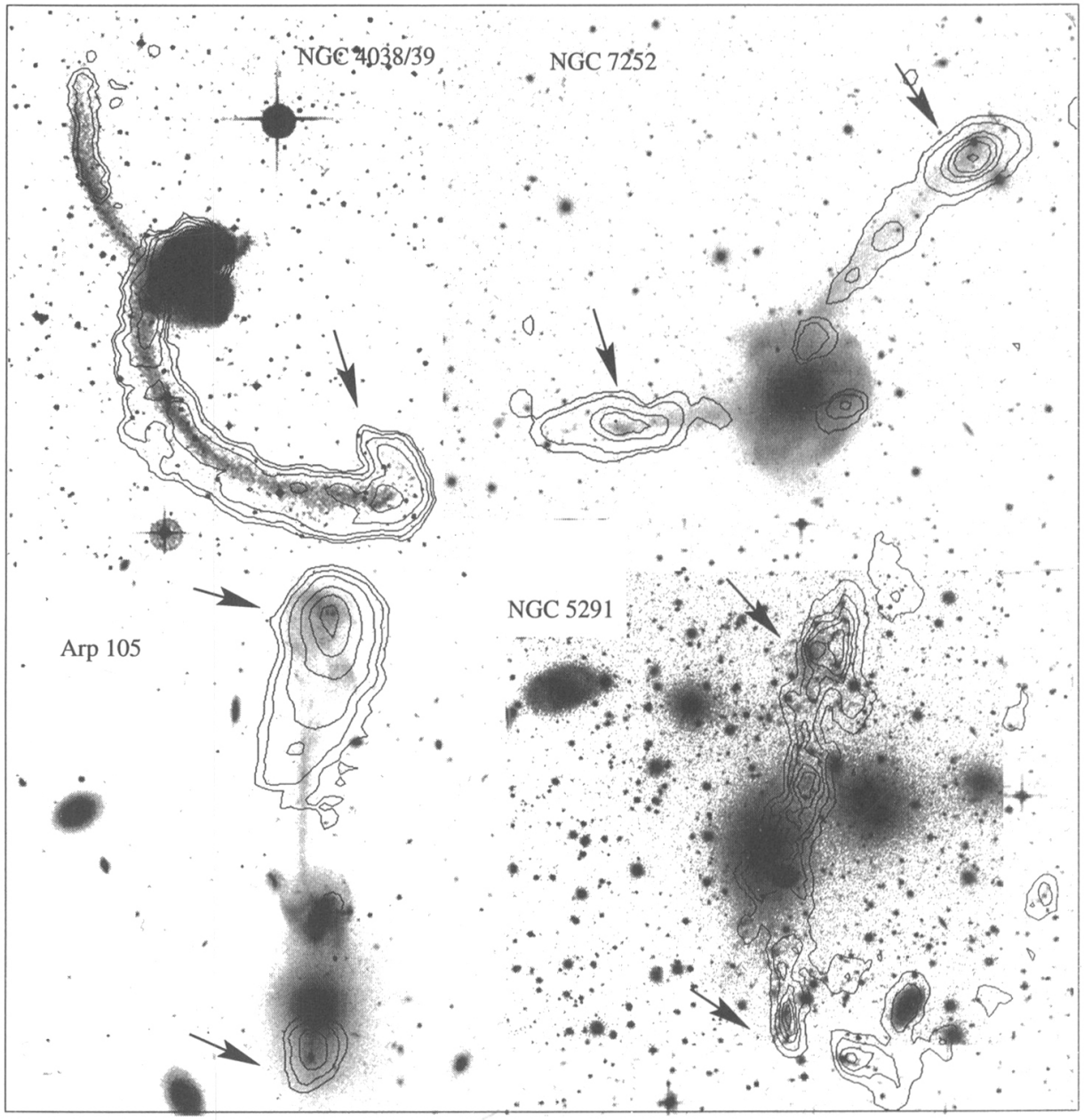

Figure 1. Tidal dwarf galaxies in four interacting systems. They are seen at the end of optical tidal tails or HI plumes and are indicated by arrows. The CCD images are from the Digital Sky Survey, Duc (1995), and Duc \& Mirabel (1997). The HI maps (black contours) are from Hibbard et al. (1997), Hibbard et al. (1994), Duc et al. (1997a), and Malphrus et al. (1997)

\subsection{STELLAR POPULATIONS}

Tidal dwarf galaxies are made of two stellar components: young stars, formed from the recent collapse of expelled HI clouds, and an older star population, also tidally pulled out from the disks of their interacting parent galaxies. Using our aperture photometry and spectroscopic data (Duc et al., 1997b), we could estimate the relative proportion of both populations and conclude that TDGs actually split into two categories 1 ) extremely young objects, forming their first generation of stars (e.g., the dwarfs around NGC 5291, Duc \& Mirabel, 1997), with high star formation rates equivalent to that observed in Blue Compact Galaxies, and 2) galaxies dominated by an old stellar population formed in the disk of their progenitors, which look like dwarf irregulars (e.g., Arp 105). In both classes of objects, the current star-forming episode, fuelled by an important HI reservoir, is younger than 10 Myrs. 


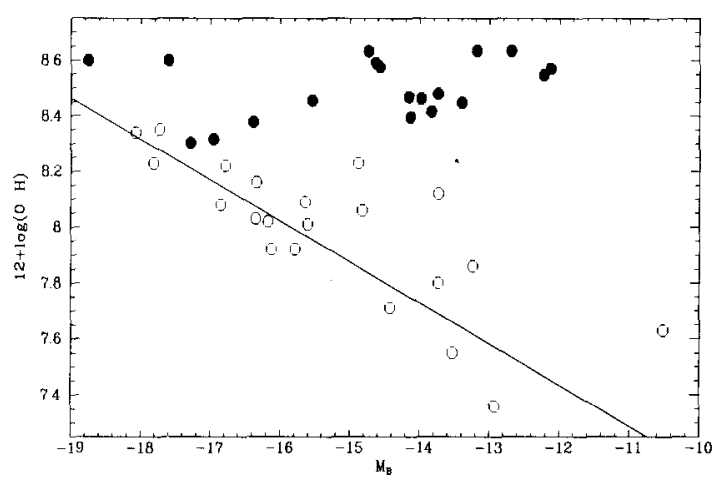

Figure 2. Oxygen abundance vs. absolute blue magnitude for our sample of tidal dwarfs (black points) and a sample of isolated dwarf galaxies (open points; from Richer \& McCall, 1995).

\subsection{METALLICITY}

Fig. 2 shows the oxygen abundance vs. absolute magnitude of a sample of TDGs and nearby dIrr. Clearly TDGs are more metal rich than classical dwarfs of the same luminosity: $Z_{\odot} / 3$ on average, a value typical of the outer regions of spirals. They actually do not follow the correlation found for field dwarf and giant galaxies between luminosity (hence mass) and metallicity. Being "recycled" objects, formed from pre-enriched material, tidal dwarfs inherited from their parents their relatively enriched interstellar medium.

\subsection{DYNAMICS AND ORIGIN}

Little is still known about the internal dynamics of tidal dwarfs. First indications are that the most massive TDGs may be gravitationally bound (Hibbard et al., 1997, Malphrus et al., 1997). Hints for rotation were found in the HI cloud associated with a TDG in Arp 105 (see Fig. 3b). Strong velocity gradients of the ionized gas were also measured (Fig. 3a). This suggests that some objects in tidal tails may already be dynamically independent. Further kinematical studies would be necessary to verify this assertion and estimate their dark matter content, predicted to be low in numerical simulations (Barnes \& Hernquist, 1992).

Models for the formation of TDGs put forward two mechanisms: a local dynamical instability in the old stellar populations of tidal tails, followed by accretion of gas (Barnes \& Hernquist, 1992) or the collapse of a supermassive cloud triggering precipitous star formation activity (Elmegreen et al., 1993). The fact that the HI component seems to dominate the mass of TDGs (Duc \& Mirabel, 1997) favors the second hypothesis. The HI potential well is large enough to trap the old star population observed in some TDGs.

\section{The fate of tidal dwarf galaxies}

Do tidal dwarf galaxies contribute significantly to the overall population of dwarf galaxies? The answer to this fundamental question depends on the frequency of tidal interactions between galaxies and on the survival time of tidal dwarfs. The latter is limited by the hostile environment of TDGs, in the vicinity of giant parent galaxies. They may fall back on their progenitors on time scales of $1 \mathrm{Gyr}$, as pointed out by Hibbard \& Mihos (1995), or be tidally disrupted. It is therefore expected that only the most massive TDGs and those that are far away enough from their progenitors will survive. This limits the number of galaxies produced to one or two per colliding system. It is therefore striking that the luminous star-forming TDGs that are found are located at the end of the optical tails, at distances from the nucleus larger than $50 \mathrm{kpc}$.

From an observational point of view, the census of TDGs is not an easy task. TDGs should obviously be searched for in the environment of interacting galaxies. Hunsberger et al. (1996) claim from the analysis of photometric data that half of the dwarf galaxies in the Hickson compact 

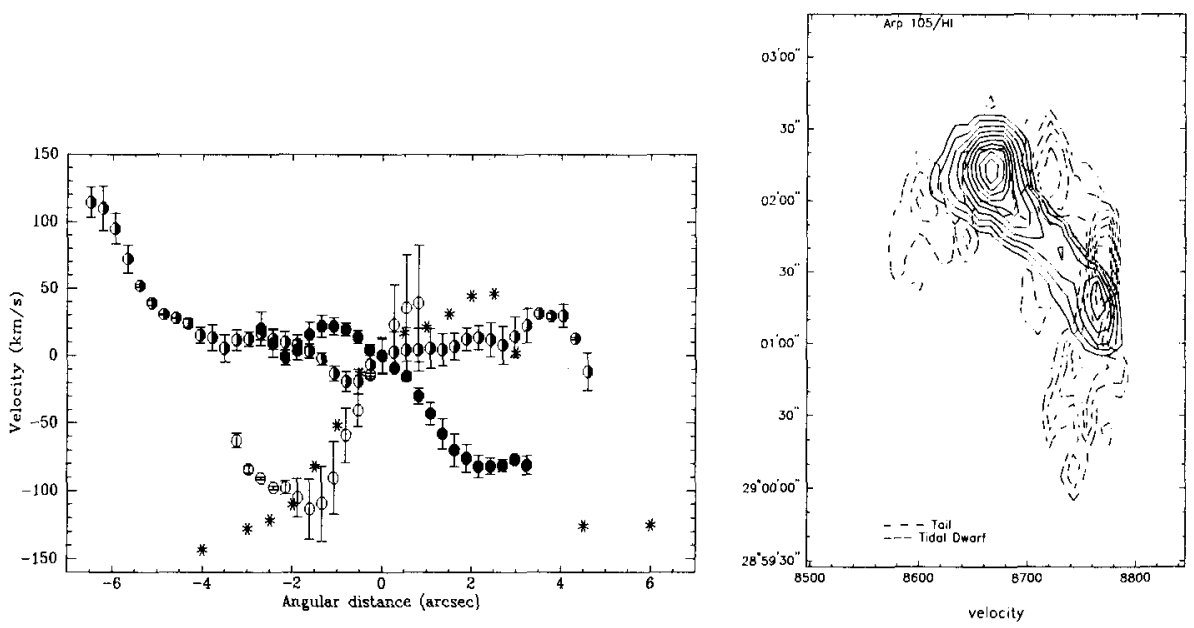

Figure 3. a) Velocity profiles of the ionized gas for several tidal dwarfs; adapted from Duc \& Mirabel (1997) b) Position-Velocity diagram of the HI northern tidal tail in Arp 105. Two components were identified: the expanding HI tidal tail (dashed contours) and a kinematically decoupled, possibly rotating, component (plain contours) associated with the forming tidal dwarf observed at the tip of the tail (see Fig. 1); adapted from Duc et al. (1997a)

groups could be of tidal origin. However, one should note that once the stellar/gaseous bridge between the parent and child galaxies has dissipated, it is difficult to re-establish a link between the two. Our study has shown that a good genetic fingerprint of TDGs is their higher metallicity. In this respect, several studies have put forward trends for dwarf galaxies in groups or clusters to be more metal rich than field dwarfs (Bothun et al. 1985; Vilchez 1995). Since the collision rate is enhanced in denser environments, it is tempting to argue that a significant fraction of dwarfs in clusters could be recycled objects. A bimodal star formation history is also a strong signature for tidal dwarfs. Evolutionary Synthesis Models simulating a burst of star formation on top of the underlying component of old galaxies reproduce well the TDG star formation history and will give constraints for their future evolution (Fritze - v. Alvensleben \& Duc, 1997).

\section{References}

Bothun, G. D., Mould, J. R., Wirth, A., and Caldwell, N. 1985, $A J, 90,697$

Duc, P.-A. 1995, Ph.D. thesis, Université Paris VI

Duc, P.-A., Brinks, E., Wink, J. E., and Mirabel, I. F. 1997a, $A \& A$, 326, 537

Duc, P.-A., Fritze-v. Alvensleben, U., and Mirabel, I. F. 1997b, in Extragalactic Astronomy in the Infrared, eds. G. Mamon, T. Thuan, and T. Tran, Editions Frontières

Duc, P.-A. and Mirabel, I. F. 1997, ABA, preprint

Elmegreen, B. G., Kaufman, M., and Thomasson, M. 1993, ApJ, 412, 90

Fritze-v.Alvensleben, U. and Duc, P.-A. 1997, in Abstract Book XXIIIrd IAU General Assembly, JD2-050P

Hibbard, J., van der Hulst, J., and Barnes, J. 1997, in preparation

Hibbard, J. E., Guhathakurta, P., van Gorkom, J. H., and Schweizer, F. 1994, AJ, 107, 67

Hibbard, J. E. and Mihos, J. C. 1995, AJ, 110, 140

Hibbard, J. E. and van Gorkom, J. H. 1996, $A J, 111,655$

Hunsberger, S. D., Charlton, J. C., and Zaritsky, D. 1996, ApJ, 462, 50

Malphrus, B., Simpson, C., Gottesman, S., and Hawarden, T. G. 1997, AJ, preprint

Mirabel, I. F., Dottori, H., and Lutz, D. 1992, $A \& A$, 256, L19

Richer, M. G. and McCall, M. L. 1995, ApJ, 445, 642

Schweizer, F. 1978, in Structure and Properties of Nearby Galaxies, eds. E. Berkhuijsen and R. Wielebinski (Reidel, Dordrecht), p. 279

Vilchez, J. M. 1995, $A J, 110,1090$ 